\title{
Infinite-time Ruin Probability of a Discrete-time Risk Model with Dependent Claims
}

\author{
Rongfei Liu, a \\ ${ }^{1}$ School of Mathematical Sciences, University of Electronic Science and Technology of China, \\ Chengdu, Sichuan 611731/West Hi-Tech Zone, China \\ aliurongfei078@gmail.com
}

Keywords: One-sided linear process; Discrete-time risk model; Heavy-tailed innovations; Asymptotic estimate

Abstract. The infinite-time ruin probability of a discrete-time risk model with dependent claims and heavy-tailed innovations is investigated in this paper. The claims are assumed to follow a one-sided linear process with independent and identically distributed (i.i.d.) innovations. Stochastic discount factors, which are independent of the innovations, and constant premium rate are taken into account. As a result, we establish an asymptotic estimate for the infinite-time ruin probability.

\section{Introduction}

Consider a discrete-time risk model as follows:

$$
U_{0}=x, U_{n}=U_{n-1} \theta_{n}^{-1}+c-X_{n}, n \geq 1 .
$$

$\mathrm{x}>0$ stands for the initial wealth of an insurer, the constant $\mathrm{c}>0$ stands for the premium rate and the nonnegative random variable (r.v.) $X_{n}$ stands for the total claim amount within period n. The investment of the surplus at time $n_{-} 1$ causes the nonnegative and stochastic discount factor $\theta_{n}$ from time $\mathrm{n}$ to time $\mathrm{n}_{-} 1$. Thus, $U_{n}$ is interpreted as the surplus of the insurer at time $\mathrm{n}$. In the terminology of Norberg[1], we call $\left\{X_{n}\right\}_{n \geq 1}$ insurance risks and call $\left\{\theta_{n}\right\}_{n \geq 1}$ financial risks.

Now we can define the infinite-time ruin probability by

$$
\Psi(x)=\mathrm{P}\left\{\min _{1 \leq m<\infty} U_{m}<0 \mid U_{0}=x\right\} .
$$

Many papers discussed the asymptotic behavior of $\Psi(x)$ under the assumption that $\left\{X_{n}\right\}_{n \geq 1}$ and $\left\{\theta_{n}\right\}_{n \geq 1}$ are two independent sequences of i.i.d. random variables (r.v.s) ([2] and [3], among many others). With the increasing complexity of insurance and reinsurance products, the assumption of independence among $\left\{X_{n}\right\}_{n \geq 1}$ is not enough to depict the real circumstances. Thus, the models of dependent insurance risks are attracting more and more attentions (for examples, [4] , [5], [6] and [7]).

In the present paper, we suppose that $\left\{X_{n}\right\}_{n \geq 1}$ and $\left\{\theta_{n}\right\}_{n \geq 1}$ are two independent sequences and $\left\{\theta_{n}\right\}_{n \geq 1}$ is a i.i.d. sequence. To depict the dependence structure of the claims, we use the following one-sided linear process to describe $\left\{X_{n}\right\}_{n \geq 1}$. Let

$$
X_{n}=\sum_{j=1}^{n} \varphi_{n-j} \varepsilon_{j}+\varphi_{n} \varepsilon_{0}, n \geq 1,
$$

where $\left\{\varphi_{n}\right\}_{n \geq 0}$ and $\varepsilon_{0}$ are nonnegative constants with $\varphi_{0}>0,\left\{\varepsilon_{n}\right\}_{n \geq 1}$ is a sequence of i.i.d. and nonnegative r.v.s with common distribution F. Assume that $\left\{\varepsilon_{n}\right\}_{n \geq 1}$, the innovations of $\left\{X_{n}\right\}_{n \geq 1}$, is independent of $\left\{\theta_{n}\right\}_{n \geq 1}$. Please see [5] and [8] for more examples of linear processes. We obtain an asymptotic estimate for $\Psi(\mathrm{x})$ when the distribution $\mathrm{F}$ belongs to the intersection of the dominated variation class $(D)$ and the long-tailed class $(L)$. 


\section{Notations and main result}

$\mathrm{C}$ represents a positive constant without relation to $\mathrm{x}$ and may vary from place to place and all limit relations are for $\mathrm{x} \rightarrow \infty$ unless stated otherwise. For two positive functions $a(\cdot)$ and $b(\cdot)$, we write $a(x) \mathrm{f} \mathrm{p} b(x)$ if $0<\liminf \mathrm{a}(\mathrm{x}) / \mathrm{b}(\mathrm{x}) \leq \lim \sup \mathrm{a}(\mathrm{x}) / \mathrm{b}(\mathrm{x})<\infty$.

By convection, an empty sum is 0 and an empty product is 1 . In order to facilitate subsequent expression, we denote

$$
\begin{aligned}
& \theta_{n, m}=\prod_{k=n}^{m} \theta_{k}, 1 \leq n \leq m \leq \infty ; Z_{\infty}=\sum_{i=1}^{\infty} \theta_{1, i} ; \\
& W_{j, \infty}=\prod_{i=j}^{\infty} \theta_{1, i} \varphi_{i-j}, 1 \leq j<\infty ; W_{0, \infty}=\sum_{i=1}^{\infty} \theta_{1, i} \varphi_{i} .
\end{aligned}
$$

A distribution $\mathrm{F}$ on $R$ has right tail function $\bar{F}$. F belongs to the dominated variation class $(D)$ if

$$
\lim \sup \frac{\bar{F}(x y)}{\bar{F}(x)}<\infty \text { for any } 0<y<1 .
$$

F belongs to the long-tailed class $(L)$ if

$$
\lim \frac{\bar{F}(x+y)}{\bar{F}(x)}=1 \text { for any } y>0 .
$$

Besides that, the upper Matuszewska index $J_{F}^{+}$and lower Matuszewska index $J_{F}^{-}$(see[9],Ch 2.1.) are used. It is well known that $J_{F}^{+}<\infty$ if $\mathrm{F} \in D$. Now, we are ready to state the main result.

Theorem 2.1. Let $\left\{X_{n}\right\}_{n \geq 1},\left\{\theta_{n}\right\}_{n \geq 1}$ be mutually independent, $\left\{X_{n}\right\}_{n \geq 1}$ be a one-sided linear process introduced in (1.3), and $\left\{\theta_{n}\right\}_{n \geq 1}$ be i.i.d. and nonnegative. If the common distribution function $F$ of the innovations $\left\{\varepsilon_{j}\right\}_{j \geq 1}$, belongs to $D \cap L, \sup _{n \geq 0} \varphi_{n}<\infty, \sum_{i=1}^{\infty} \mathrm{E}_{1}^{p i}<\infty$ for some $J_{F}^{+}<p \leq 1$ and $\sum_{j=1}^{\infty} \mathrm{E} W_{j, \infty}^{p_{i}}<\infty, i=1,2$ for some $0<p_{1}<J_{F}^{-} \leq J_{F}^{+}<p_{2}<p$, then it holds that

$$
\lim \left|\frac{\Psi(x)}{\sum_{j=1}^{\infty} \mathrm{P}\left\{\varepsilon_{j} W_{j, \infty}>x\right\}}-1\right|=0 .
$$

\section{Proof of the main result}

\section{Some lemmas}

By Proposition 2.2.1 in [9], for a distribution $\mathrm{F} \in D$, it holds that

$$
x^{-p}=o(\bar{F}(x)) \text { for any } p>J_{F}^{+} .
$$

From Lemma 3.2 in [10], Lemma 3 in [6] and Lemma 4.1.2 in [3], we have three lemmas.

Lemma 3.1. Let $X$ and $Y$ be two independent and nonnegative random variables, where $X$ is distributed by $\mathrm{F}$. If $\mathrm{F} \in D$, then for any fixed $\delta>0$ and $p>J_{F}^{+}$, there exists a positive constant $\mathrm{C}$ without relation to $\delta$ and $\mathrm{Y}$ such that for all large $\mathrm{x}$,

$$
\mathrm{P}(X Y>\delta x \mid Y) \leq C \bar{F}(x)\left[\delta^{-p} Y^{p}+1_{[Y<\delta]}\right] .
$$

Lemma 3.2. Let $X$ and $Y$ be two independent and nonnegative random variables, where $X$ is distributed by $\mathrm{F}$. If $\mathrm{F} \in D$, then for any fixed $\delta>0$ and $0<p_{1}<J_{F}^{-} \leq J_{F}^{+}<p_{2}<\infty$, there exists a positive constant $\mathrm{C}$ without relation to $\delta$ and $\mathrm{Y}$ such that for all large $\mathrm{x}$,

$$
\mathrm{P}(X Y>\delta x \mid Y) \leq C \bar{F}(x)\left[\delta^{-p_{1}} Y^{p_{1}}+\delta^{-p_{2}} Y^{p_{2}}\right] .
$$

Lemma 3.3. Let $X$ and $Y$ be two independent and nonnegative random variables, where $X$ is distributed by $\mathrm{F}$ and $\mathrm{Y}$ is nondegenerate at 0 . If $\mathrm{F} \in D \cap L$ and $E Y^{p}<\infty$ for some $p>J_{F}^{+}$, then the distribution of $X Y$ belongs to $D \cap L$ and $\mathrm{P}(X Y>x) \mathrm{f} \mathrm{p} \bar{F}(x)$.

The following lemma will play crucial role in the proof of Theorem 2.1. 
Lemma 3.4. Under the conditions of Theorem 2.1 , for any $c_{0} \geq 0$, it holds that

$$
\lim \left|\frac{\mathrm{P}\left\{\sum_{j=0}^{\infty} \varepsilon_{j} W_{j, \infty}-c_{0} Z_{\infty}>x\right\}}{\sum_{j=1}^{\infty} \mathrm{P}\left\{\varepsilon_{j} W_{j, \infty}>x\right\}}-1\right|=0 .
$$

Proof. Under the conditions of Theorem 2.1 and by $\mathrm{Cr}$ inequality, we can get

$$
\begin{aligned}
& \mathrm{E} Z_{\infty}^{p}=\mathrm{E}\left[\left(\sum_{i=1}^{\infty} \theta_{1, i}\right)^{p}\right] \leq C\left(\sum_{i=1}^{\infty} \mathrm{E}_{1}{ }^{p i}\right)<\infty, \\
& \mathrm{E} W_{j, \infty}^{p} \leq\left(\sup _{n \geq 0} \varphi_{n}\right)^{p} \mathrm{E}\left[\left(\sum_{i=1}^{\infty} \theta_{1, i}\right)^{p}\right]<\infty, j \geq 0 .
\end{aligned}
$$

Firstly, we deal with the upper bound. For any fixed $0<\mathrm{L}<\infty$ and $\mathrm{k}$, we can obtain

$$
\begin{aligned}
& P\left\{\sum_{j=0}^{\infty} \varepsilon_{j} W_{j, \infty}-c_{0} Z_{\infty}>x\right\} \\
& \leq P\left\{\sum_{j=0}^{\infty} \varepsilon_{j} W_{j, \infty}>x\right\} \\
& \leq P\left\{\bigcup_{j=0}^{\infty}\left\{\varepsilon_{j} W_{j, \infty}>x-L\right\}\right\}+P\left\{\sum_{j=0}^{\infty} \varepsilon_{j} W_{j, \infty}>x,\left.\right|_{j=1} ^{k}\left\{\varepsilon_{j} W_{j, \infty} \leq x-L\right\}\right\} \\
& :=I_{1}+I_{2} .
\end{aligned}
$$

By Lemma 3.3 and (3.4), the distribution of $\varepsilon_{j} W_{j, \infty}$ belongs to $D \cap L$ and

$$
P\left\{\varepsilon_{j} W_{j, \infty}\right\} \text { f p } \bar{F}(x) \text {. }
$$

Then, by Chebyshev's inequality and (3.1), we can get

$$
\begin{aligned}
& I_{1} \leq \sum_{j=1}^{\infty} P\left\{\varepsilon_{j} W_{j, \infty}>x-L\right\}+P\left\{\varepsilon_{0} W_{0, \infty}>x-L\right\} \\
& \leq(1+o(1)) \sum_{j=1}^{\infty} P\left\{\varepsilon_{j} W_{j, \infty}>x\right\}+\frac{\varepsilon_{0}^{p} \mathrm{E} W_{0, \infty}^{p}}{(x-L)^{p}} \\
& \leq(1+o(1)) \sum_{j=1}^{\infty} P\left\{\varepsilon_{j} W_{j, \infty}>x\right\}+o(\bar{F}(x)) .
\end{aligned}
$$

By Lemma 3.1, we can obtain that for all large $\mathrm{x}$ and any fixed $\mathrm{k}$,

$$
\begin{aligned}
& I_{2} \leq P\left\{\sum_{j=0}^{\infty} \varepsilon_{j} W_{j, \infty}>x,\left.\right|_{j=1} ^{k}\left\{\varepsilon_{j} W_{j, \infty} \leq x-L\right\}, \bigcup_{i=1}^{k}\left\{\varepsilon_{i} W_{i, \infty}>\frac{x}{k}\right\}\right\} \\
& \leq \sum_{i=1}^{k} P\left\{\varepsilon_{i} W_{i, \infty}>\frac{x}{k}, \sum_{j=0, j \neq i}^{\infty} \varepsilon_{j} W_{j, \infty}>L\right\} \\
& \leq \sum_{i=1}^{k} C \bar{F}(x) \mathrm{E}\left[k^{p} W_{i, \infty}^{p} 1_{\left\{\sum_{j=0, j \neq i}^{\infty} \varepsilon_{j} W_{j, \infty}>L\right\}}+1_{\left\{\sum_{j=0, j \neq i}^{\infty} \varepsilon_{j} W_{j, \infty}>L\right.}\right] .
\end{aligned}
$$

Then, by (3.4), there exists $\mathrm{L}^{*}$ such that for any fixed $\mathrm{L} \geq \mathrm{L}^{*}$,

$$
I_{2} \leq o(\bar{F}(x))
$$

Combining (3.5), (3.7) and (3.8), we can get

$$
\begin{aligned}
& P\left\{\sum_{j=0}^{\infty} \varepsilon_{j} W_{j, \infty}-c_{0} Z_{\infty}>x\right\} \\
& \leq(1+o(1)) \sum_{j=1}^{\infty} P\left\{\varepsilon_{j} W_{j, \infty}>x\right\}+o(\bar{F}(x)) \\
& \leq(1+o(1)) \sum_{j=1}^{\infty} P\left\{\varepsilon_{j} W_{j, \infty}>x\right\},
\end{aligned}
$$

where we used (3.6) in the last step.

Secondly, we deal with the lower bound. For any fixed $0<D<\infty$ and $\mathrm{k}$, we have 


$$
\begin{aligned}
& P\left\{\sum_{j=0}^{\infty} \varepsilon_{j} W_{j, \infty}-c_{0} Z_{\infty}>x\right\} \\
& \geq P\left\{\sum_{j=1}^{k} \varepsilon_{j} W_{j, \infty}-c_{0} Z_{\infty}>x\right\} \\
& \geq P\left\{\bigcup_{j=1}^{k}\left\{\varepsilon_{j} W_{j, \infty}>x+c_{0} Z_{\infty}\right\}\right\} \\
& \geq \sum_{j=1}^{k} P\left\{\varepsilon_{j} W_{j, \infty}>x+c_{0} Z_{\infty}, Z_{\infty} \leq D\right\}-\sum_{i=1}^{k} \sum_{1 \leq j \leq k, j \neq i} P\left\{\varepsilon_{i} W_{i, \infty}>x, \varepsilon_{j} W_{j, \infty}>x\right\} \\
& \geq \sum_{j=1}^{k} P\left\{\varepsilon_{j} W_{j, \infty}>x+c_{0} D\right\}-\sum_{j=1}^{k} P\left\{\varepsilon_{j} W_{j, \infty}>x+c_{0} D, Z_{\infty}>D\right\} \\
& -\sum_{i=1}^{k} \sum_{1 \leq j \leq k, j \neq i} P\left\{\varepsilon_{i} W_{i, \infty}>x, \varepsilon_{j} W_{j, \infty}>x\right\} \\
& :=L_{1}-L_{2}-L_{3} .
\end{aligned}
$$

Because the distribution of $\varepsilon_{j} W_{j, \infty}$ belongs to $D \cap L$, we can get

$$
L_{1} \geq(1-o(1)) \sum_{j=1}^{k} P\left\{\varepsilon_{j} W_{j, \infty}>x\right\} .
$$

By Lemma 3.1, we can obtain that for all large $\mathrm{x}$,

$$
L_{2} \leq \sum_{j=1}^{k} P\left\{\varepsilon_{j} W_{j, \infty}>x, Z_{\infty}>D\right\} \leq \sum_{j=1}^{k} C \bar{F}(x) \mathrm{E}\left[W_{j, \infty}^{p} 1_{\left\{Z_{\infty}>D\right\}}+1_{\left\{Z_{\infty}>D\right\}}\right] .
$$

Then, by (3.4), there exists $\mathrm{D}^{*}$ such that for any fixed $\mathrm{D} \geq \mathrm{D}^{*}$,

By Lemma 3.1 and (3.4), we can get

$$
L_{2} \leq o(\bar{F}(x)) \text {. }
$$

$$
L_{3} \leq \sum_{i=1}^{k} \sum_{1 \leq j \leq k, j \neq i} C \bar{F}(x) \mathrm{E}\left[W_{i, \infty}^{p} 1_{\left\{\varepsilon_{j} W_{j, \infty}>x\right\}}+1_{\left\{\varepsilon_{j} W_{j, \infty}>x\right\}}\right] \leq o(\bar{F}(x)) .
$$

Hence, combining (3.9)-(3.12), we obtain

$$
\begin{aligned}
& P\left\{\sum_{j=0}^{\infty} \varepsilon_{j} W_{j, \infty}-c_{0} Z_{\infty}>x\right\} \\
& \geq(1-o(1)) \sum_{j=1}^{k} P\left\{\varepsilon_{j} W_{j, \infty}>x\right\}-o(\bar{F}(x)) \\
& =(1-o(1))\left(\sum_{j=1}^{\infty}-\sum_{j=k+1}^{\infty}\right) P\left\{\varepsilon_{j} W_{j, \infty}>x\right\}-o(\bar{F}(x)) .
\end{aligned}
$$

By Lemma 3.2 and condition, there exists $\mathrm{k}^{*}$ such that for $\mathrm{k} \geq \mathrm{k}^{*}$ and any $0<p_{1}<J_{F}^{-} \leq J_{F}^{+}<p_{2}<p$,

$$
\sum_{j=k+1}^{\infty} P\left\{\varepsilon_{j} W_{j, \infty}>x\right\} \leq C \bar{F}(x) \sum_{j=k+1}^{\infty} E\left[W_{j, \infty}^{p_{1}}+W_{j, \infty}^{p_{2}}\right] \leq o(\bar{F}(x)) .
$$

Thus, we can derive

$$
\begin{aligned}
& P\left\{\sum_{j=0}^{\infty} \varepsilon_{j} W_{j, \infty}-c_{0} Z_{\infty}>x\right\} \\
& \geq(1-o(1)) \sum_{j=1}^{\infty} P\left\{\varepsilon_{j} W_{j, \infty}>x\right\}-o(\bar{F}(x)) \\
& \geq(1-o(1)) \sum_{j=1}^{\infty} P\left\{\varepsilon_{j} W_{j, \infty}>x\right\},
\end{aligned}
$$

where we used (3.6) in the last step.

\section{Proof of Theorem 2.1}

Proof. From (1.1), we get that for $\mathrm{n} \geq 1$,

$$
U_{n}=x \theta_{1, n}^{-1}+\sum_{i=1}^{n}\left[\theta_{i+1, n}^{-1}\left(c-X_{i}\right)\right] .
$$

Substituting (3.13), (1.3) into (1.2) and rewriting the expression, we can derive 


$$
\begin{aligned}
& \Psi(x)=P\left\{\min _{0 \leq m<\infty} U_{m} \theta_{1, m}<0\right\} \\
& =P\left\{\max _{0 \leq m<\infty} \sum_{i=1}^{m}\left[\theta_{1, i}\left(X_{i}-c\right)\right]>x\right\} \\
& =P\left\{\max _{0 \leq m<\infty}\left(\sum_{j=0}^{m} \varepsilon_{j} W_{j, m}-c Z_{m}\right)>x\right\} .
\end{aligned}
$$

It is clear that

$$
P\left\{\sum_{j=0}^{\infty} \varepsilon_{j} W_{j, \infty}-c Z_{\infty}>x\right\} \leq \Psi(x) \leq P\left\{\sum_{j=0}^{\infty} \varepsilon_{j} W_{j, \infty}>x\right\} .
$$

Then, by Lemma 3.4, we can get (2.1).

\section{References}

[1]Norberg R. Ruin problems with assets and liabilities of diffusion type. Stochastic Processes and their Applications, 1999; 81(2): 255-269.

[2] Tang Q, Tsitsiashvili G. Randomly weighted sums of subexponential random variables with application to ruin theory. Extremes, 2003; 6: 171-188.

[3] Wang D, Tang Q. Tail probabilities of randomly weighted sums of random variables with dominated variation. Stochastic Models, 2006; 22: 253-272.

[4] Bowers NL, Gerber HU, Hickman JC, Jones DA, Nesbitt CJ.Actuarial Mathematics, $2^{\text {nd }}$ ed.The Society of Actuaries: Schaumburg, IL, 1997.

[5] Gerber HU. Ruin theory in the linear model. Insurance: Mathematics and Economics ,1982; 1: 213-217.

[6] Guo F,Wang D. Uniform asymptotic estimates for ruin probabilities of renewal risk models with exponential Lévy process investment returns and dependent claims. Applied Stochastic Models in Business and Industry, 2013; 29: 295-313.

[7] Peng J, Huang J, Wang D. The ruin probability of a disctete-time risk model with a onesided linear claim process. Communications in Statistics-Theory and Methods, 2011; 40: $\quad$ 4387-4399.

[8] Yang H, Zhang L. Martingale method for ruin probability in an autoregressive model with constant interest rate. Probability in the Engineering and Informational Sciences, 2003; 17: 183-198.

[9] Bingham NH, Goldie CM, Teugels JL. Regular Variation. Cambridge University Press:Cambridge, 1987.

[10] Heyde CC,Wang D. Finite-time ruin probability with an exponential Lévy process investment return and heavy-tailed claims. Advances in Applied Probability, 2009; 41: 206-224. 